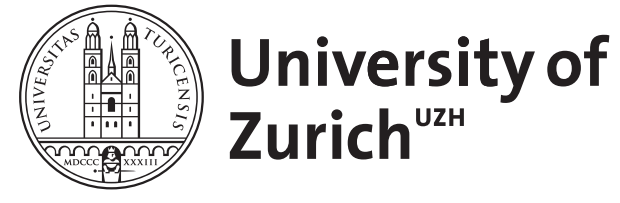

\title{
From the rodent spinal cord injury model to human application: Promises
} and challenges

Dietz, V ; Schwab, M E

DOI: https://doi.org/10.1089/neu.2016.4513

Posted at the Zurich Open Repository and Archive, University of Zurich ZORA URL: https://doi.org/10.5167/uzh-129447

Journal Article

Accepted Version

Originally published at:

Dietz, V; Schwab, M E (2017). From the rodent spinal cord injury model to human application: Promises and challenges. Journal of Neurotrauma, 34(9):1826-1830.

DOI: https://doi.org/10.1089/neu.2016.4513 
J Neurotrauma, Opinion paper

From the rodent spinal cord injury model to human application: Promises and challenges

Volker Dietz (1) and Martin E. Schwab (2), University (1), University and ETH (2) Zürich,

Switzerland

Address for correspondence:

Prof. em. Dr Volker Dietz, FRCP

Spinal Cord Injury Center

University Hospital Balgrist

Forchstr.340

CH 8008 Zürich

vdietz@paralab.balgrist.ch 
Research approaches to improve outcome of sensori-motor functions after a spinal cord injury (SCI) concern first, experimental therapies that enhance neuronal and tissue repair in animals; some of these approaches are on the way to translation into clinical trials. A second line of research is based on the enhancement of neuroplasticity by a functional training being a main (and also classical) driver to improve outcome of function after SCI. This research is based on both, basic research in animals, especially rodents, as well as in human beings. In the last 30 years, animal and human research made several important discoveries, creating insights and concepts that greatly have advanced the field. However, not all problems are solved yet, and novel ones also arose. The focus of this opinion paper will be on the actual promises and challenges for the translation of new concepts and therapeutic approaches from the rodent SCI model to the application in human SCI.

\section{Basic research in animals}

Animal models are central to our understanding of the pathophysiology and the repair processes after SCI. Central topics over the last 30 years were the characterization of growth inhibitory and growth promoting factors in the central nervous system (CNS) tissue, the restricted neuronal growth capacity of adult CNS neurons, inflammatory reactions at lesion sites, scar formation, neuroprotection and attempts to bridge the lesion, as well as myelin repair. Stimulation of the spinal cord combined with the application of dopaminergic drugs and an intense training was another approach, based on the inherent, activity-enhanced plasticity of neuronal circuits within the spinal cord below the lesion. Anatomical, physiological and behavioral read-outs were used. Several approaches showed promising results in particular in terms of an improved functional outcome. For a few of these experimental therapies, clinical trials in spinal cord injured patients are currently on-going or planned.

The following promises and challenges arise in the translation from bench to bedside: 


\section{Promises}

- The discovery of specific growth inhibitory factors in the adult CNS, in particular the myelin enriched membrane protein Nogo-A as well as the scar and perineuronal net associated chondroitin sulfate proteoglycans, triggered experiments where these factors were neutralized (e.g. by antibodies), destroyed (e.g. by chondroitinase), genetically deleted or down knocked, or inactivated by interfering with their receptors or downstream intracellular signaling pathways (for reviews). ${ }^{1}{ }^{3}$ In many studies from different laboratories, enhanced neuritic sprouting was seen from lesioned (regenerative sprouting) as well as spared (compensatory sprouting) fibers in the damaged spinal cord and brain. Long distance regeneration of small proportions of fibers through remaining tissue bridges was also reproducibly observed. Anti-Nogo-A antibody therapy is in clinical trials at present for SCI, amyotrophic lateral sclerosis (ALS) and multiple sclerosis (MS). The second messenger RhoA blocker Cethrin/VX210, which shows a similar effect profile, is also in Phase II trials at present.

- Enhancement of neurite growth and regeneration can be induced on the neuronal level by stimulating the mTOR pathway (e.g. by suppression of its inhibitor Phosphatase and tensin homolog PTEN, stimulation of the cytokine signaling pathway, or the cAMP pathway. ${ }^{4,5}$ The functional consequence of this growth stimulation in the spinal cord is not well known yet. In the crushed optic nerve, however, regeneration of a few fibers up to the pretectum and superior colliculus with the restoration of light responses was reported. ${ }^{6}$ No clinical trials were started yet using these approaches.

\footnotetext{
-The stabilization of the axonal cytoskeleton, in particular the microtubules was recently shown to significantly enhance regenerative tract sprouting and growth in the injured rodent spinal cord. ${ }^{7,8}$ Interestingly, quite low concentrations of Taxol or epothiloneB exert these effects in vitro and in vivo, conditions that could be readily translated into clinical tests.
} 
-Bridging the lesion site by cellular transplants has been tried over many years with variable success. Implanted materials often get walled off by scarring astrocytes, but Schwann cells, ${ }^{9}$ peripheral nerve grafts ${ }^{10}$ or olfactory ensheathing cells ${ }^{11}$ can integrate into the spinal cord tissue and form bridges for regenerating axons. The exact conditions for reproducible bridge formation are not well known, and the variability in these experiments between labs and publications is high. Injections of autologous olfactory ensheathing cells (OEC) were tried in chronic human SCI. Neither negative nor beneficial effects were found in individuals with motor complete SCI. ${ }^{12}$ Also results from a larger group of individuals examined in China did not show signs of motor recovery $\left(\mathrm{cf}^{13}\right)$ On the other hand, a single case with positive effects of these transplanted cells was recently reported in a Polish study. ${ }^{14}$

During last years the most successful bridging study consisted of a combination of multiple peripheral nerve grafts with fibrin glue, neural stem cells (with a strong growth potential), and a neurotrophic growth factor. ${ }^{15}$ Serotoneric axons grew through these bridges and reinnervated adjacent spinal cord segments. While stem cell derived axons reached the lower spinal cord as well as the brain, the functional (or malfunctional) role of these fibers remains unclear up to now.

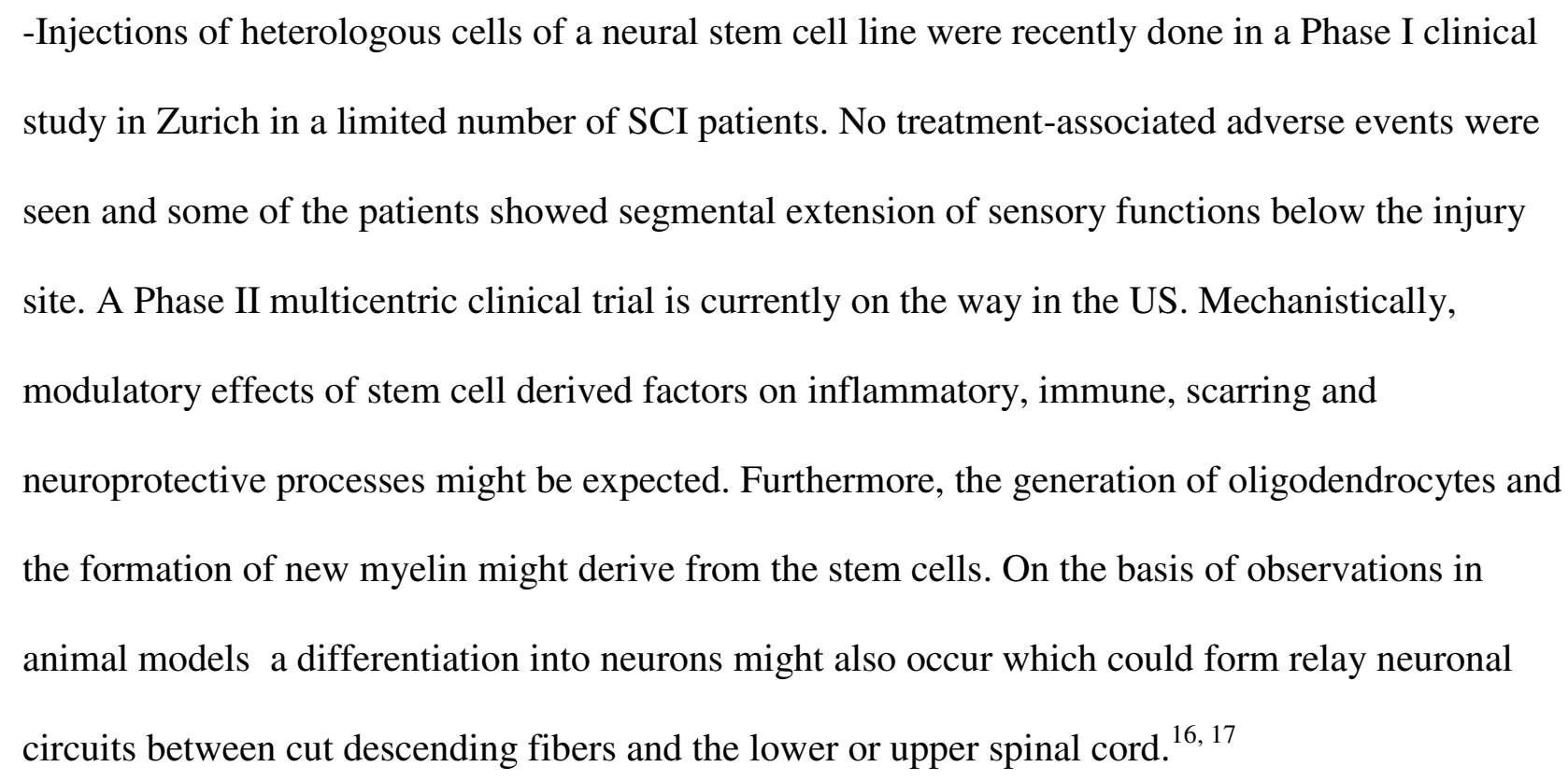
study in Zurich in a limited number of SCI patients. No treatment-associated adverse events were seen and some of the patients showed segmental extension of sensory functions below the injury site. A Phase II multicentric clinical trial is currently on the way in the US. Mechanistically, modulatory effects of stem cell derived factors on inflammatory, immune, scarring and neuroprotective processes might be expected. Furthermore, the generation of oligodendrocytes and the formation of new myelin might derive from the stem cells. On the basis of observations in animal models a differentiation into neurons might also occur which could form relay neuronal circuits between cut descending fibers and the lower or upper spinal cord. ${ }^{16,17}$ 
-Training experiments performed originally in spinal transected cats showed that regular stepping as well as standing can be trained in the lumbar spinal cord completely separated from the brain. ${ }^{18,}$ ${ }^{19}$ The underlying concept was that the locomotor pattern generator within the lumbar spinal cord becomes activated by external stimuli. ${ }^{20}$ In the early nineties, this locomotor training approach was successfully translated to spinal cord injured humans. ${ }^{21,22}$ Training-induced locomotor activity could be demonstrated even in patients with a complete SCI. ${ }^{23}$ Treadmill training, assisted manually by physiotherapists or by robots, is now part of routine rehabilitation programs in many SCI rehabilitation centers. Thus this training approach represents a typical example of highly successful clinical translation of findings and concepts from basic science.

\begin{abstract}
-Direct epidural stimulation of the lumbar spinal cord, sometimes in combination with intrathecal drug administration of serotonergic and/or dopaminergic agonists, results in enhanced mobility on treadmills in the rodent SCI model. ${ }^{24-26}$ This combined approach is still in an experimental stage. Interestingly, clonidine was shown to improve walking ability in the cat with a transected spinal cord ${ }^{27}$. Its intrathecal application in human SCI resulted in incomplete injury in inconsistent effects $^{28}$,in complete injury in a flaccid paresis. ${ }^{21}$.
\end{abstract}

-The stimulation part of this approach was recently translated to human SCI with interesting results. $^{29,30}$ A combination of intense training and epidural spinal cord stimulation allowed motor complete SCI patients the performance of small voluntarily controlled foot and leg movements, as well as improved standing (however not stepping movements). The approach is limited to severely injured, paralysed SCI patients who have some spared axons running through the damaged part of the spinal cord. Thus, in these patients stimulation elevates the excitability of spinal neuronal circuits, or of spared descending connections to a level wheresome neuronal circuits can function and 'voluntary' commands can become minimally effective. How generally applicable these 
therapeutic interventions will be and what their benefit for the daily life of patients could be, will be seen in the future once a larger number of SCI patients have been treated.

\section{Challenges}

- The functional read-outs in animal SCI models usually focus on the recovery of sensori-motor functions following an intervention. According to a query in SCI patients, ${ }^{31}$ these functions have lower priority for humans suffering an SCI; highest priorities are given to bladder control and sexual functions. Only a few research groups work on adequate models for these functions at present. ${ }^{32-34}$ A better understanding leading to good animal models and read-outs of e.g. bladder dysreflexia, blood pressure dysregulation and the connectivity underlying sexual functions is urgently needed.

\footnotetext{
- Most interventions use thoracic lesions in rodent models with the aim to induce recovery of hindlimb locomotor function. For tetraplegic/quadriplegic, i.e. cervical spinal cord injured, patients with their loss of independence and quality of life, successful interventions to improve hand function and breathing would be of utmost importance. Cervical injuries are complicated by the fact of a combined damage of central and peripheral nervous structures (i.e. motoneurons and spinal roots), the latter contributes to up to $50 \%$ of paresis ${ }^{35}$ (for review see. ${ }^{36}$ ) The rare use of cervical injury models in animals, in particular when applying large lesions, is also related to ethical issues and the difficulty to keep these animals in a good overall health status.
}

-In a large number of animal studies, the lesions used are transections, - which are rare in human SCI patients. The typical lesion in humans is a contusion, sometimes a laceration, extending over 2 to 3 segments, with bleedings and necrosis. Cysts and scars also form after transections, but the lesions are better defined and less variable, - which is the main reason why basic researchers usually prefer them and why weight drop contusions are rarely in use. 


\begin{abstract}
-Many interventions, such as the growth promoting Nogo antibodies or mTOR manipulations require preserved tissue bridges at the lesion site to allow axonal regeneration through the damaged part of the spinal cord. Such bridges are present in all incomplete SCI patients, but, interestingly, also in some clinically complete SCI patients.
\end{abstract}

\begin{abstract}
- Description of the lesion extent, in particular also with regard to spared parts of the white matter and of specific tracts, is often performed in animal studies (based mostly on postmortem histology), but is almost impossible in SCI patients (however see Cohen-Adad and WheelerKingshott $2014^{37}$ ). This is due to the difficulty to image patients with long imaging time protocols early after the accident, and the presence of metal implants as soon as the spinal cord is surgically stabilized. In addition, tracing and reconstruction of specific axonal tracts is routine in many animal studies, but missing with the current imaging technology in humans.
\end{abstract}

\begin{abstract}
-For some important sequels of a human SCI such as spastic movement disorder or neurogenic pain no adequate animal model exists. Spinal shock, which last for 4-6 weeks in acute SCI patients, is only short and less pronounced in animals. Muscle spasms develop in SCI rats, but reflect cramps rather than spastic muscle tone and are not yet well studied. ${ }^{38}$
\end{abstract}

\footnotetext{
- In rodent models, therapeutic interventions are applied usually early or immediately after the spinal cord lesion. In acute human traumatic SCI, life saving treatments have the highest priority. Furthermore, for clinical trials the intervention has to be approved by the patient in a full conscious state. Therefore, a novel experimental intervention can hardly be applied earlier than 2 to 3 weeks after trauma. Such a delay could be a critical factor responsible for differences seen in the effects of an intervention when comparing animal models and human SCI.
} 


\title{
Clinical problems requiring basic animal research
}

Basic animal research is not only needed to promote the progress in the field of regeneration.

Clinical researchers also require basic animal research to answer important questions arising from clinical observations in neurorehabilitation. For example:

-During locomotor training of chronic motor complete SCI subjects an exhaustion of leg muscle and spinal motoneuron activity occurs. ${ }^{39}$ Why does this dysfunction of neuronal spinal circuits below the level of lesion in SCI patients develop? There are rodent studies indicating an undirected neuroplasticity as a possible mechanism. ${ }^{40}$ In which way can this neuronal dysfunction be avoided or inversed? The proper functioning of neuronal circuits below the level of lesion is the prerequisite for a successful regeneration.

\begin{abstract}
-Neuroplasticity is supposed to be the neuronal substrate for the effects of functional training during neurorehabiliation. Its elements, - fiber growth and formation of new neuronal circuits, activation of 'silent connections', strengthening of pre-existing synapses, - need to be dissected. A key question is in which way these processes can be influenced and what roles are played by inflammatory and degenerating processes on the one hand, and training-induced neuronal activity on the other one. What are the individual limitations of neuroplasticity for an improved outcome?
\end{abstract}

-The newly discovered mechanism of neural coupling as manifested in cooperative hand movements ${ }^{41}$ should be modelled in animals to investigate the underlying neuronal circuitries. This could help to optimize their responses to training of hand function and to experimental interventions in tetraplegic patients.

- The exploration of pain mechanisms should lead to measures that help to avoid the development of neurogenic pain and find a causal treatment of pain following an SCI. ${ }^{42}$

\section{Basic research in humans}




\begin{abstract}
Translational research concerns not only the translation of concepts and therapies from animal models to human conditions. Basic research performed in humans frequently also serves as the basis for novel rehabilitation approaches in patients with a CNS damage, e.g. after an SCI.
\end{abstract}

\title{
Promises
}

- Locomotor treadmill training was introduced into the clinic 25 years ago, originally based on cat experiments. ${ }^{18}$ Subsequent human experiments showed that specific physiological receptor activation, such as load and hip joint related receptors, is required during such a training to achieve a physiological leg muscle activity and, consequently, an improved locomotor function. ${ }^{43}$ Optimal protocols for the duration of locomotor training, the distribution over the training day, the degree of progressive loading and additional physiological parameters are still required.

- Locomotor training on the treadmill is physically exhausting and requires the assistance of usually two physiotherapists and is therefore limited in time. Consequently, the idea came up to replace the manual by a robotic assistance. The prototype 'Lokomat' was designed by Gery Colombo in the research lab of the Spinal Cord Injury Center in Zürich (for review ${ }^{44}$ ) and subsequently improved and brought to market by the company Hocoma. Together with an upper limb training device of a group at MIT, these training robots triggered the development of robot assisted rehabilitation. New generations of these training robots include feedback controlled assistas-needed systems and virtual reality set-ups to optimally motivate the patients. SCI, stroke, Parkinson's disease and pediatric brain damage are interesting fields of application of this technology. Today besides the Lokomat several exoskeletons are available to assist stepping movements but require more input from the patient ${ }^{45}$ Still, however, several questions arise that can only be answered by human experiments: (i) What is the optimal approach and what are the 
limits to exploit neuroplasticity by such devices. (ii) What are the best cues to enhance training effects (e.g. movement velocity, duration of training). Presently such information is not available.

-Questions such as individual limits of recovery of function due to neuroplasticity can hardly be answered from animal experiments. Robot assisted functional movements have to be designed on the basis of physiological requirements (e.g. activation of specific relevant receptors) in order to achieve an appropriate limb muscle activation and, consequently, to become beneficial. This requires a close cooperation of engineers with basic and clinical scientists.

-The effect of virtual reality in the training of functional movements: Only a few studies exist on the effects of a non-motivating vs. a highly motivating virtual reality set-up combined with robotassisted rehabilitation programs. ${ }^{46}$

-The effect of specific neural mechanisms underlying physiological hand movements: It could be shown by electrophysiological and imaging studies that a task-specific neural coupling mechanism underlies cooperative (e.g. opening a bottle) compared to bimanual separate hand movements. This coupling is reflected in an involvement of both hemispheres in the control of each hand. ${ }^{41}$ Consequently, after a cervical SCI or a stroke both hemispheres become involved in the control of the paretic hands during such cooperative movements. ${ }^{47}$ For the rehabilitation of upper limb movements this has the consequence that a training of cooperative hand movements required during a number of daily life activities, eventually in combination with an exclusive training of the affected arm/hand (Constraint Induced Movement Therapy) can improve outcome of hand function in subjects suffering a CNS damage. ${ }^{47}$

\section{Challenges}


-The observations made on the neuronal mechanisms underlying functional movement disorders have to a large part remain phenomenological. Basic animal experiments are required to get insight into neuronal mechanisms and pathways involved and to prove their specific contributions to a lesion-induced deficit and/or recovery process.

-In human studies no direct access is possible to spinal and supraspinal neuronal mechanisms underlying, for example, the development of pain after an SCI. Animal models in different species that allow cellular and neuronal circuitry-based insights but also translation to the human level are needed.

- For most interventions, such as repair strategies, clinical trials can only be initiated after careful and extensive preclinical studies which reduce the risk of adverse side effects to a minimum. A direct application of a novel intervention to humans is rarely possible. Such preclinical studies are usually costly, require certified good laboratory practice (GLP) and, therefore, often exceed the capabilities of academic laboratories. Such hurdles have often been responsible for the slow pace of translation from bench to bedside, also in the SCI field.

\section{Conclusions}

Novel technological or pharmacological approaches to exploit and enhance neuroplasticity and regeneration potential of the nervous system have to be developed in a close, multi-way interaction between basic and clinical neuroscientists, occupational and physiotherapists as well as engineers. These cooperations can and have to become optimized in the field of SCI research to accelerate translations of novel basic animal and human science concepts into therapies for the benefit of the spinal cord injured patients. 


\begin{abstract}
Epilogue
VD (neurologist, former head of the University of Zurich Spinal Cord Injury Center) and MES (neurobiologist) conducted joint research projects over more than 20 years in Zürich (ETH and University). This included a translational study of spinal cord regeneration (Nogo-A antibody phase 1 study) from the rodent model (MS) to human SCI (VD). We are convinced that only by a close cooperation between basic and clinical scientists basic research achievements can successfully be translated to the human condition.
\end{abstract}




\section{References}

1. Schwab, M.E. (2010). Functions of Nogo proteins and their receptors in the nervous system. Nat Rev Neurosci 11, 799-811.

2. Schwab, M.E. and Strittmatter, S.M. (2014). Nogo limits neural plasticity and recovery from injury. Current Opinion in Neurobiology 27, 53-60.

3. Fawcett, J.W. (2009). Recovery from spinal cord injury: regeneration, plasticity and rehabilitation. Brain 132, 1417-1418.

4. Mar, F.M., Bonni, A. and Sousa, M.M. (2014). Cell intrinsic control of axon regeneration. EMBO Reports 15, 254-263.

5. Yang, P. and Yang, Z. (2012). Enhancing intrinsic growth capacity promotes adult CNS regeneration. Journal of the Neurological Sciences 312, 1-6.

6. de Lima, S., Koriyama, Y., Kurimoto, T., Oliveira, J.T., Yin, Y., Li, Y., Gilbert, H.-Y., Fagiolini, M., Martinez, A.M.B. and Benowitz, L. (2012). Full-length axon regeneration in the adult mouse optic nerve and partial recovery of simple visual behaviors. Proceedings of the National Academy of Sciences 109, 9149-9154.

7. Ruschel, J., Hellal, F., Flynn, K.C., Dupraz, S., Elliott, D.A., Tedeschi, A., Bates, M., Sliwinski, C., Brook, G., Dobrindt, K., Peitz, M., Brüstle, O., Norenberg, M.D., Blesch, A., Weidner, N., Bunge, M.B., Bixby, J.L. and Bradke, F. (2015). Systemic administration of epothilone B promotes axon regeneration after spinal cord injury. Science 348, 347-352.

8. Hellal, F., Hurtado, A., Ruschel, J., Flynn, K.C., Laskowski, C.J., Umlauf, M., Kapitein, L.C., Strikis, D., Lemmon, V., Bixby, J., Hoogenraad, C.C. and Bradke, F. (2011). Microtubule Stabilization Reduces Scarring and Causes Axon Regeneration After Spinal Cord Injury. Science $331,928-931$.

9. Bunge, M.B. and Pearse, D.D. (2003). Transplantation strategies to promote repair of the injured spinal cord. J Rehabil Res Dev 40, 55-62. 
10. Cheng, H., Cao, Y. and Olson, L. (1996). Spinal cord repair in adult paraplegic rats: partial restoration of hind limb function. Science 273, 510-513.

11. Raisman, G., Barnett, S.C. and Ramón-Cueto, A. (2012). Chapter 33 - Repair of central nervous system lesions by transplantation of olfactory ensheathing cells. In: Handbook of Clinical Neurology. Joost, V., John, W.M. (eds). Elsevier, pps. 541-549.

12. Mackay-Sim, A., Feron, F., Cochrane, J., Bassingthwaighte, L., Bayliss, C., Davies, W., Fronek, P., Gray, C., Kerr, G., Licina, P., Nowitzke, A., Perry, C., Silburn, P.A., Urquhart, S. and Geraghty, T. (2008). Autologous olfactory ensheathing cell transplantation in human paraplegia: a 3-year clinical trial. Brain 131, 2376-2386.

13. Dobkin, B.H., Curt, A. and Guest, J. (2006). Cellular transplants in China: observational study from the largest human experiment in chronic spinal cord injury. Neurorehabil Neural Repair 20, $5-13$.

14. Tabakow, P., Raisman, G., Fortuna, W., Czyz, M., Huber, J., Li, D., Szewczyk, P., Okurowski, S., Miedzybrodzki, R., Czapiga, B., Salomon, B., Halon, A., Li, Y., Lipiec, J., Kulczyk, A. and Jarmundowicz, W. (2014). Functional Regeneration of Supraspinal Connections in a Patient With Transected Spinal Cord Following Transplantation of Bulbar Olfactory Ensheathing Cells With Peripheral Nerve Bridging. Cell Transplantation 23, 1631-1655.

15. Lu, P., Wang, Y., Graham, L., McHale, K., Gao, M., Wu, D., Brock, J., Blesch, A., Rosenzweig, Ephron S., Havton, Leif A., Zheng, B., Conner, James M., Marsala, M. and Tuszynski, Mark H. (2012). Long-Distance Growth and Connectivity of Neural Stem Cells after Severe Spinal Cord Injury. Cell 150, 1264-1273.

16. Cummings, B.J., Uchida, N., Tamaki, S.J., Salazar, D.L., Hooshmand, M., Summers, R., Gage, F.H. and Anderson, A.J. (2005). Human neural stem cells differentiate and promote locomotor recovery in spinal cord-injured mice. PNAS 102 14069-14074.

17. Lu, P., Woodruff, G., Wang, Y., Graham, L., Hunt, M., Wu, D., Boehle, E., Ahmad, R., Poplawski, G., Brock, J., Goldstein, Lawrence S.B. and Tuszynski, Mark H. (2014). Long- 
Distance Axonal Growth from Human Induced Pluripotent Stem Cells after Spinal Cord Injury. Neuron 83, 789-796.

18. Barbeau, H. and Rossignol, S. (1994). Enhancement of locomotor recovery following spinal cord injury. Curr Opin Neurol 7, 517-524.

19. Lovely, R.G., Gregor, R.J., Roy, R.R. and Edgerton, V.R. (1990). Weight-bearing hindlimb stepping in treadmill-exercised adult spinal cats. Brain Research 514, 206-218.

20. Grillner, S. and Matsushima, T. (1991). The neural network underlying locomotion in Lamprey-synaptic and cellular mechanisms. Neuron 7, 1-15.

21. Dietz, V., Colombo, G., Jensen, L. and Baumgartner, L. (1995). Locomotor capacity of spinal cord in paraplegic patients. Ann Neurol 37, 574-582.

22. Wernig, A., Nanassy, A. and Muller, S. (1999). Laufband (treadmill) therapy in incomplete paraplegia and tetraplegia. J Neurotrauma 16, 719-726.

23. Dietz, V., Colombo, G. and Jensen, L. (1994). Locomotor activity in spinal man. Lancet 344, 1260-1263.

24. Courtine, G., Gerasimenko, Y., van den Brand, R., Yew, A., Musienko, P., Zhong, H., Song, B., Ao, Y., Ichiyama, R.M., Lavrov, I., Roy, R.R., Sofroniew, M.V. and Edgerton, V.R. (2009). Transformation of nonfunctional spinal circuits into functional states after the loss of brain input. Nature neuroscience 12, 1333-1342.

25. Edgerton, V.R., Courtine, G., Gerasimenko, Y.P., Lavrov, I., Ichiyama, R.M., Fong, A.J., Cai, L.L., Otoshi, C.K., Tillakaratne, N.J., Burdick, J.W. and Roy, R.R. (2008). Training locomotor networks. Brain Res Rev 57, 241-254.

26. Edgerton, V.R., Tillakaratne, N.J., Bigbee, A.J., de Leon, R.D. and Roy, R.R. (2004).

Plasticity of the spinal neural circuitry after injury. Annu Rev Neurosci 27, 145-167.

27. Forssberg, H. and Grillner, S. (1973). The locomotion of the acute spinal cat injected with clonidine i.v. Brain Res 50, 184-186. 
28. Remy-Neris, O., Barbeau, H., Daniel, O., Boiteau, F. and Bussel, B. (1999). Effects of intrathecal clonidine injection on spinal reflexes and human locomotion in incomplete paraplegic subjects. Exp Brain Res 129, 433-440.

29. Harkema, S., Gerasimenko, Y., Hodes, J., Burdick, J., Angeli, C., Chen, Y., Ferreira, C., Willhite, A., Rejc, E., Grossman, R.G. and Edgerton, V.R. (2011). Effect of epidural stimulation of the lumbosacral spinal cord on voluntary movement, standing, and assisted stepping after motor complete paraplegia: a case study. Lancet 377, 1938-1947.

30. Angeli, C.A., Edgerton, V.R., Gerasimenko, Y.P. and Harkema, S.J. (2014). Altering spinal cord excitability enables voluntary movements after chronic complete paralysis in humans. Brain 137, 1394-1409.

31. Anderson, K.D. (2004). Targeting recovery: priorities of the spinal cord-injured population. J Neurotrauma 21, 1371-1383.

32. Schneider, M.P., Hughes, F.M., Engmann, A.K., Purves, J.T., Kasper, H., Tedaldi, M., Spruill, L.S., Gullo, M., Schwab, M.E. and Kessler, T.M. (2015). A novel urodynamic model for lower urinary tract assessment in awake rats. BJU International 115, 8-15.

33. Nout, Y.S., Schmidt, M.H., Tovar, C.A., Culp, E., Beattie, M.S. and Bresnahan, J.C. (2005). Telemetric Monitoring of Corpus Spongiosum Penis Pressure in Conscious Rats for Assessment of Micturition and Sexual Function following Spinal Cord Contusion Injury. Journal of Neurotrauma 22, 429-441.

34. Lee, Y.-S., Lin, C.-Y., Jiang, H.-H., DePaul, M., Lin, V.W. and Silver, J. (2013). Nerve Regeneration Restores Supraspinal Control of Bladder Function after Complete Spinal Cord Injury. The Journal of Neuroscience 33, 10591-10606.

35. Thomas, C.K., Zaidner, E.Y., Calancie, B., Broton, J.G. and Bigland-Ritchie, B.R. (1997). Muscle weakness, paralysis, and atrophy after human cervical spinal cord injury. Exp Neurol 148, 414-423. 
36. Dietz, V. and Fouad, K. (2014). Restoration of sensorimotor functions after spinal cord injury. Brain 137, 654-667.

37. Cohen-Adad, J. and Wheeler-Kingshott, C. (2014). Quantitative MRI of the spinal cord. Academic Press.

38. Gonzenbach, R.R., Gasser, P., Zorner, B., Hochreutener, E., Dietz, V. and Schwab, M.E. (2010). Nogo-A antibodies and training reduce muscle spasms in spinal cord-injured rats. Ann Neurol 68, 48-57.

39. Dietz, V., Grillner, S., Trepp, A., Hubli, M. and Bolliger, M. (2009). Changes in spinal reflex and locomotor activity after a complete spinal cord injury: a common mechanism? Brain 132, 2196-2205.

40. Beauparlant, J., van den Brand, R., Barraud, Q., Friedli, L., Musienko, P., Dietz, V. and Courtine, G. (2013). Undirected compensatory plasticity contributes to neuronal dysfunction after severe spinal cord injury. Brain 136, 3347-3361.

41. Dietz, V., Macauda, G., Schrafl-Altermatt, M., Wirz, M., Kloter, E. and Michels, L. (2015). Neural coupling of cooperative hand movements: a reflex and FMRI study. Cereb Cortex 25, 948958.

42. Cragg, J.J., Haefeli, J., Jutzeler, C.R., Rohrich, F., Weidner, N., Saur, M., Maier, D.D., Kalke, Y.B., Schuld, C., Curt, A. and Kramer, J.K. (2016). Effects of Pain and Pain Management on Motor Recovery of Spinal Cord-Injured Patients: A Longitudinal Study. Neurorehabil Neural Repair.

43. Dietz, V., Muller, R. and Colombo, G. (2002). Locomotor activity in spinal man: significance of afferent input from joint and load receptors. Brain 125, 2626-2634.

44. Riener, R., Lünenburger, L., Maier, I.C., Colombo, G. and Dietz, V. (2010). Locomotor Training in Subjects with Sensori-Motor Deficits: An Overview of the Robotic Gait Orthosis Lokomat. J Healthcare Engin 1, 197-216. 
45. Wu, M. and Landry, J.M. (2012). Lower Extremity Flexible Assist Devices for Locomotion. In: Neurorehabilitation Technology. Springer, pps. 361-378.

46. Brütsch, K., Schuler, T., Koenig, A., Zimmerli, L., Mérillat, S., Lünenburger, L., Riener, R., Jäncke, L. and Meyer-Heim, A. (2010). Influence of virtual reality soccer game on walking performance in robotic assisted gait training for children. Journal of NeuroEngineering and Rehabilitation 7, 15-15.

47. Schrafl-Altermatt, M. and Dietz, V. (2016). Cooperative hand movements in stroke patients: Neural reorganization. Clinical Neurophysiology 127, 748-754. 